\title{
Emerging Trends in Various Fields with Systems Biology Approach
}

\author{
Siva Kishore Nandikolla ${ }^{1 *}$, Mahaboobbi Shaik ${ }^{2}$, Satya Varali $\mathbf{M}^{3}$ and Ramya Seelam ${ }^{4}$
}

${ }^{1}$ Department of Biochemistry and Bioinformatics, GITAM University, Visakhapatnam, India

${ }^{2}$ Department of Biotechnology, Andhra University, Visakhapatnam, India

${ }^{3}$ Department of Human Genetics, Andhra University, Visakhapatnam, India

${ }^{4}$ Department of Biotechnology, Satyabhama University, Chennai, India

\begin{abstract}
Systems biology explains how higher level properties of complex biological systems arise from the interactions among their parts. This requires a combination of concepts from many disciplines, including biology, computer science, applied mathematics, physics and engineering. The importance and its various implementations in various fields like proteomics, genomics, metabolomics, docking studies etc., recent works and advancements are portrayed in this review article.
\end{abstract}

\section{Introduction}

Systems biology means understanding complex biological systems with the integration of experimental and computational research [1]. Systems biology describes a number of trends in bioscience research, and draws importance on those trends. Some describe systems biology as a biology-based inter-disciplinary subject that focuses on complex interactions in biological systems with new perspective of holism instead of reduction. The motive of systems biology is the modeling and discovery of emergent properties of a system whose theoretical description is only possible only by using systems biology technology. These generally involve cell signaling networks [2]. Systems biology has its roots in the quantitative modeling of enzyme kinetics, mathematical modeling of population growth and the simulations developed to study neurophysiology, control theory and cybernetics.

\section{Systems Biology is also associated with disciplines like}

Phenomics-isastudyconcerned with themeasurementofphenomes, the biochemical and physical traits of organisms with respect to their change in response to environmental influences and genetic mutation. Genomics - the study of the organisms genome is called genomics. This field includes derives the entire DNA sequence of organisms and finescale genetic mapping efforts.

Epigenetics - study of heritable changes in cellular phenotype or gene expression caused by mechanisms other than changes happen in DNA sequence

Transcriptomics - the set of all RNA molecules is called transcriptome, which includes mRNA, rRNA, tRNA, and other noncoding RNA. This study of transcriptome is called Transcriptomics.

Interactomics - it is a combined subject of bioinformatics and biology which deals with both the interactions and the consequences of protein-protein interactions, and other cellular molecules [3].

Interferomics - the study of post-transcriptomic vanges and pretranslatomic changes.

Translatomics or Proteomics - is the study of proteins, in particular of their structures and functions $[4,5]$. Proteins are essential for living organisms, as they are the main components of the metabolic pathways of cells.

Metabolomics - study of the unique chemical fingerprints which leaves behind specific cellular processes, the study of these profiles of small-molecule metabolite is called Metabolomics [6].
Glycomics - the study of all glycans of a given cell type or organism. This comes under glycobiology.

Lipidomics - the large-scale study of networks and pathways of cellular lipids in biological systems [7,8].

NeuroElectroDynamics - the study of the dynamics and interaction of electrical charges in the brain [9].

Fluxomics - a mathematical method for analysing metabolism is known as fluxomics. Knowledge of concentrations of metabolites or the details of enzyme kinetics of the system is not required for this mathematical method.

Biomics - is the biological knowledge of Biomes, and the processing of that information, like ecological communities of plants, animals, and micro organisms comes under biomics.

Biological function can be understood if we are able to integrate all relevant information at multiple levels of organization to recreate dynamic interactions. Mathematical and computational models together explain the underlying complex interacting non-linear processes in biological function which cannot be recreated by experimental observation [10].

System Biology studies paved a huge role in various fields. The recent developments in some the fields are noted below.

\section{Proteomics}

There is rising death of humans worldwide because of tuberculosis. The current sequencing of the Mycobacterium tuberculosis genome gave way for the development of new vaccines and the design of new drugs [11]. Based on sequence similarity accurate annotation for genes in newly sequenced genomes are made [12]. About 250 hypothetical proteins of Mycobacterium tuberculosis functions were predicted using

*Corresponding author: Siva Kishore Nandikolla, Department of Biochemistry and Bioinformatics, GITAM University, India, E-mail: sivakishoren@gmail.com

Received April 15, 2011; Accepted May 20, 2011; Published May 23, 2011

Citation: Siva Kishore N, Mahaboobbi S, Satya Varali M, Ramya S (2011) Emerging Trends in Various Fields with Systems Biology Approach. J Comput Sci Syst Biol S13. doi:10.4172/0974-7230.S13-004

Copyright: ( 2011 Siva Kishore N, et al. This is an open-access article distributed under the terms of the Creative Commons Attribution License,which permits unrestricted use, distribution, and reproduction in any medium, provided the original author and source are credited. 
Bioinformatics web tools, BLAST, INTERPROSCAN, PFAM [13] and COGs [14]. Spirulina platensis crystal structure for Phycocyanin (PDB ID 1GH0) contains 24 chains named from 1GH0A to $1 \mathrm{GH} 0 \mathrm{X}$. Alternate chains consisted of same sequence however, the odd chains (1GH0A, 1GH0C, 1GH0E... 1GH0W) and even chains (1GH0B, 1GH0D, 1GH0F... 1GHOX) contained 162 and 172 amino acid residues respectively in similar pattern. Sequence comparison revealed 100 BLAST hits and phylogenetic tree was drawn for alternate chains. Structure comparisons were examined by VAST showed residues of alternate full chains from 1 to 162 and from 1 to 172 residues to contain 1323 structure neighbors. 1628 structure neighbors were found for the phycobilisome domain family which is the major accessory light- 1628 harvesting complexes of cyanobacteria and red algae [15]. Regarding how different organisms are related to each other and tracing their evolutionary relationship for their common ancestor remains controversial. Phylogenetic analysis can be done based on either protein sequences or nucleic acid. Analysis of loops in elongation factors EF1A using novel informative characteristic which was called "loops" method is applied. Each kingdom showed differences in the number of loops and their location in the three EF1A domains and can be taken as imprint of molecular evolution [16]. The cation-p interaction is an important, general force for molecular recognition in biological receptors. Secondary structure of the cation-p involving residues shows that, Arg and Lys prefers to be in strand. Among the $\mathrm{p}$ residues, Phe prefer to be in coil, Tyr prefers to be in strand and Trp prefer to be in helix. Among the cation-p interacting residues Arg and Lys were in the exposed regions. The contribution of stabilizing residues in the cation-p interactions was analyzed. The comparison between the conventional and nonconventional interactions in the data set, clearly depict the significance of cation-p interaction in the stability of therapeutic proteins. [17]. Hepatitis B virus genome size is $3.215 \mathrm{~kb}$. To predict the epitopes from 7 putative proteins through polymerase, large-S- and middle -S- Protein, S and X- protein, Precore/Core Protein, Core and E- antigen, immunoinformatics tools are used. Epitopes might be helpful for designing peptide based subunit vaccine against Hepatitis B virus [18]. Genomic projects provided a wide amount of information that still requires be analyzing and interpreting. It is not possible without development of effective computational techniques. For analyzing proteomes a tool is developed and implemented through the CGI and PERL that can simulate the two-dimensional electrophoresis from a whole genome [19].

Proteins interacting directly with each other have similar conditions and involve in the same cell processes. The same families of disease phenotypes are lead by mutations in genes. Priority is given for positional candidate genes of complex diseases which uses the protein-protein interaction (PPI) information. But this method is often considered general for specific diseases [20]. Proteins are important components of the cellular level. Comparative analysis between prokaryotes and eukaryotes non-membrane proteins (nMPs) made to determine the bias nature in amino acids. It revealed that 'Ala' is the dominant amino acid in prokaryotic nMPs while 'Lys, Ser and Cys' in eukaryotic nMPs [21]. Delta hepatitis is pandemic disease which is caused by Hepatitis delta Virus (HDV) an RNA virus [22]. HDV causes either co-infection or super infection with Hepatitis B virus. Small delta antigen protein of HDV is obligatory for replication of virus. It plays a vital role in virus life cycle; it can be a suitable drug target. 3D structure of protein is very important for the rational design. 3-D modeling of small delta antigen protein was done by using GenThreader [23] followed by modeller9v7. The predicted 3-D structure was performede using PROCHECK
[24], Anolea, Gromos96 and Swisspdb-viewer tools. To study surface features and functional binding pockets in protein, CASTp was used. The resulting 3-D model can be developed as novel inhibitor to cure diseases with [25]. Antibiotic discovery aimed at conventional targets such as proteins and nucleic acids faces challenges from mutations and antibiotic resistance. The BioCyc interactive metabolic pathway maps and Pathway Tools software were used to corroborate our finding. Ten metabolites are identified as potential candidates for developing novel antibiotics. These metabolites are Lipid II, meso-diaminopimelate, pantothenate, shikimate, biotin, L-aspartyl-4-phosphate, dTDP-aL-rhamnose, UDP Dgalacto-1,4-furanose, des-N-acetyl mycothiol, and Siroheme. The selection criteria, analysis of metabolic pathways, and the potential role of each of the ten metabolites in therapeutic intervention as broadspectrum antibiotics with emphasis on $M$. tuberculosis is made [26]. Damage analysis in metabolic pathways is one of the most highlighted fields in systems biology area. Metabolic errors are studied with respect to inherited enzyme deficiencies. The deletion of a path may change the end product of a reaction and can give rise to disorders. Various statistical methods have been employed to determine the damages in the metabolic pathway. One such method is graph analysis technique. The wet lab results may be interpreted using statistics. Metabolomics can be performed using flux based analysis, fourier -transformation, Lp and power laws [27].

\section{Genomics}

Polymerase chain reaction (PCR) is an enzymatic reaction which uses simple, predictable and accepted principles. Nucleic acid amplification, that are initially present in small quantities, provides a powerful tool for assaying nucleic acids. Efficiency and sensitivity of the PCR depends on the efficiency of the primers that are used for the amplification of a respective gene [28]. Matarray is a microarray image processing and data analysis package, where quality scores are assigned for every spot that reflects the variability of the data acquired from each spot. A new advancement in Matarray is that the quality scores are impregnated as weights in the statistical evaluation and data mining of microarray data. Significant utilization of control clones are given in ratios 1: 30 to 30:1. The quality weighted statistics are more accurate in gene expression measurements, sensitive detection of their changes with significantly lower type II error rates [29]. A novel statistical model

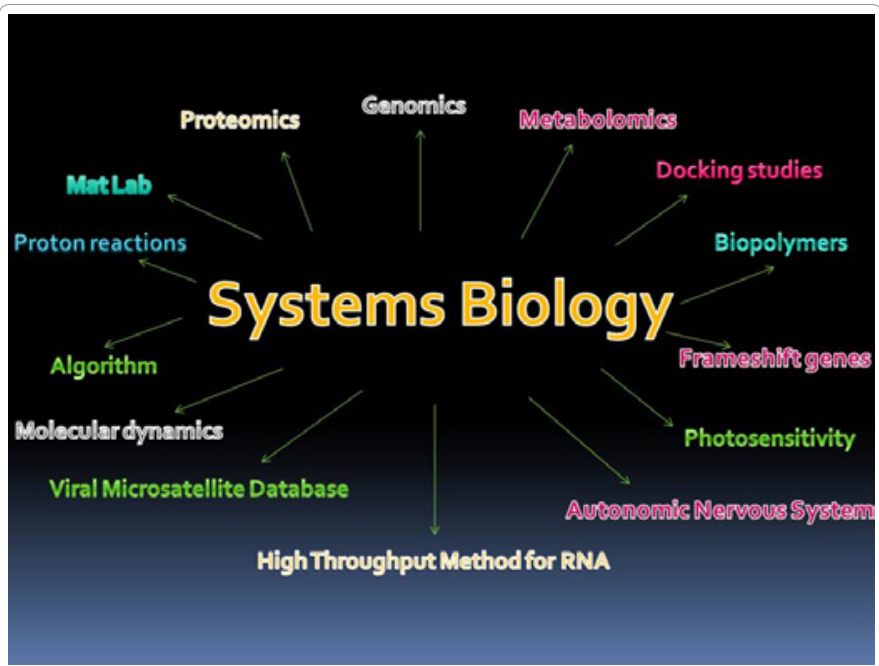

Figure 1: Overall View of Applications of Systems Biology. 
for cancer gene identification is made by integrating the gene mutation hypothesis of cancer formation into the mixture model framework. Within this framework, genetic interactions of DNA sequences (or haplotypes) between host and cancer genes responsible for cancer risk are defined The model is formulated within the maximum likelihood context and implemented with the EM algorithm. The implications of this model for cancer gene identification can be discussed [30]. Study of yeast's transcription regulatory network in association with gene expression profiles shows that $S R$ and $D R$ probabilities are significantly associated with the distribution of strong co-expression. It is 100 fold more probable to observe co-expression when $P(S R) » 0.5$ for a random perturbation of 3 transcription factors (TFs). The relationship between the model derived probabilities and other graph-theoretic measures used to analyse biological networks [31]. The process of sequencing in bio-macromolecules is determined and conditioned not only through biochemical, but also through information principles and cybernetics [32]. In gene therapy the integration process of the viral DNA genome into the host cell genome is a necessary step for virus integration. Before, retrovirus integration was thought to be random and the chance of activating a gene was considered rare. Depending on whether the provirus integrates into or in the vicinity of genes (Transcription Start Sites, TSS), normal transcription can be enhanced or disrupted thus inducing oncogenic mutations. This is called "insertional mutagenesis". Developing statistical procedures often lead to a unique definition of CIS rather than a "problem related" definition [33]. Genomics helped the identification of microbial virulence factors, which is important for vaccine design, especially for live attenuated vaccines. Several methods have been applied to find virulence genes like DNA microarrays, In Vivo Expression Technology (IVET), Signature-Tagged Mutagenesis (STM), Differential Fluorescence Induction (DFI). ORFs can hide some true virulence factors, all of these imaginary proteins from various respiratory diseases and their predicted biological functions by a careful analysis of each one [34]. The genome sequence information is essential to understand the function of extensive arrangements of genes. It is significant to combine all sequence information in a precise database to provide an efficient manner of sequence similarity search. Oryza sativa (japonica cultivar-group) species is an important cereal and model monocot. A matrix frequency for genetic code analysis helps in the study of complete genome residues. A new method of Chaos Game Representation produces the objects possessing selfsimilar structure [35]. The performance of most methods for cancer diagnosis using gene expression data greatly depends on careful model selection. Linear regression as a constrained 11-norm minimization problem greatly alleviate its sensitivity to outliers, and hence the name 11 least square. The numerical experiment shows that 11 least square can match the best performance achieved by support vector machines (SVMs) with careful model selection [36]. Humans have been carrying unwanted viral gene segments since many years and reports suggests that approximately $3-8 \%$ of the human genome has been comprised of viral DNA. Various viral sequences from NCBI were downloaded and scanned against complete genome of Homo sapiens for the presence of possible viral inserts in human genome. The results from the computational analysis revealed that dengue virus segments inserted in the intron regions of human genome and exon region insertions were observed with polio and simian enterovirus. The alignments which show $>25-30$ residues, $90-100 \%$ identities and the sequences located in the exon regions were considered [37]. Hepatitis B viral sequence was downloaded from NCBI Tax Browser and scanned against complete genome of Homo sapiens for the presence of possible viral inserts in human genome. The alignments which showed more than 25-30 residues, 90-100\% identities and localization in the exon regions were only considered. The results from the computational analysis revealed that Hepatitis B virus resulted in viral segment inserted in the exon region of human genome Single nucleotide polymorphism is one of the genetic markers which are most widely used in genetic association studies of a population. SNPs are DNA sequence variations are caused when a single nucleotide ( $\mathrm{A}, \mathrm{T}, \mathrm{C}$, or $\mathrm{G})$ in the genome sequence is altered. SNPs which are in coding sequence are more vulnerable to alterations in the biological function of a protein. The SNPs found in this region and its linkage disequilibrium analysis to find out the effect of SNPs found and there correlation. Present computational analysis is to find out SNPs in the chromosome1 [38]. The dbEST section of Genbank has 1467 ESTs of Prosopis juliflora are utilized for the improvement of genetic microsatellite markers (SSRs). The analysis of the putative unigenes has been shown to have most abundant motif of $\mathrm{A} / \mathrm{T}$ followed by dinucleotide $\mathrm{AG} / \mathrm{CT}$ and trinucleotide repeat AAG/CTT. EST-derived SNPs are becoming the resources for the development of SNP markers. The relative rates of development of the high throughput computational methods for the detection of SNPs (Single Nucleotide Polymorphism) and small indels (insertion / deletion) has wide application range in the field of the molecular markers [39].

\section{Metabolomics}

Metabolic networks symmetry has not been systematically investigated. In directed graph symmetry was introduced and an algorithm to calculate it in directed and disconnected graphs was developed. Several indices to measure the degree of symmetry are calculated and compared them with random networks. These metabolic networks in KEGG and BioCyc databases are generally symmetric and in particular locally symmetric. The symmetry in metabolic networks is distinctly higher than that in random networks. The metabolic network symmetry can generate the functional redundancy, increase the effectiveness and play an important role in network structure, function and evolution [40]. Staphylococcus aureus is one of the most important and studied gram positive bacterial strains, which have a great potential to infect human beings as well as other mammals. This antibiotic resistance strain, lead to development of the new antibiotics or drug molecules which can kill or suppress the growth of Staphylococcus aureus. A comparative analysis of metabolic pathways of the host Homo sapiens and the pathogen S. aureus gave the e-value threshold cut-off to 0.005 . A total of 235 enzyme sequences were identified, which are non homologous to Homo sapiens protein sequences. Further PASUB analysis Results showed that about $52.5 \%$ enzymes are found to be in the cytoplasm, $13.5 \%$ enzymes are found to be in extracellular, $6.7 \%$ enzymes are plasma membrane protein and $27.1 \%$ enzymes are given no positive prediction [41]. The genome sequence information is required to understand the function of of genes. It is suggestive to combine all sequence information in a precise database to provide an efficient manner of sequence similarity search [42]. A noticeable success was made on designing optimized systems that increase the productivity and minimize the raw materials costs for valuable metabolites $[43,44]$. It is a new addition to the 'omics' field, metabolomics is evolving its own computational infrastructure and assessing its own computational needs $[45,46]$.

\section{Docking studies}

Structure-based lead optimization approaches are playing a great role in the process of drug-discovery. The two docking programs (Arguslab [47] and Surflex [48]), for virtual database screening 
performance are studied. Surflex is commercial package while Arguslab is free package. The problem of scoring and docking flexible compounds is the sterical capable of docking into a rigid conformation of the receptor [49]. Various proteins play important roles in hypertension and a number of plants have been tested for their efficacy in modulating hypertension. Angiotensin 1-converting enzyme, renin and extracellular regulated kinase 2(ERK2) proteins, respectively, has major role in hypertension and therefore protein - ligand interaction studies were performed on 266 compounds from different parts of 7 plants (Allium sativum, Coriandrum sativum, Dacus carota, Murrayya koneigii, Eucalyptus globus, Calendula officinalis and Lycopersicon esculentum). Analysis was conducted using GOLD (Genetic Optimisation for Ligand Docking) software as docking program and the molecules drawn in ISIS Draw software are energy minimized using cosmic - optimize 3D module of Tsar (Tools for structure activity relationships) software [50]. Malaria is the most important parasitic disease in humans, with transmission occurring in over 100 countries with a population of three billion people. This is done by inhibiting the enzyme, dihydrofolate reductase. The side effects are needed to improve drugs. Conformational analysis and geometry optimization of Proguanil was performed using Argus Lab \& Hex software. The most dockable position for the drug to interact with the receptor was found to be with analog 2 having energy $-9.56 \mathrm{~K}$.cal/mole using Argus Lab and -201.92 K.cal/mole using HEX Tool. So Proguanil Analog 2 sketched using Chemsketch is detected with more significant energy values in both softwares and probable lead molecules [51]. Pathogenic bacteria use the needle shaped Type III secretion system to inject effector proteins into the host cell. The SopB effector protein of Salmonella mediates invasion by evading the host immune response. Being a phosphoinositide phosphatase, it synthesizes phospholipids at the host cell membrane, after targeting host cell ubiquitin. Ubiquitination of SopB are known to control the biological activity of SopB at the plasma membrane. The conserved Glu 228 of SopB protein was predicted to be involved in imparting a electronegative potential in the ligand binding site. The ubiquitin molecule docked with SopB of V.vulnificus had Leu8 for binding interaction and recognition which was found to be similar to the ubiquitin-human polymerase complex. The host immune response was predicted to be targeted by SopB effector in V.vulnificus by altering the ubiquitin pathway [52].

\section{Biopolymers}

For the long range structure of biopolymers entropy plays an important role. The lattice model entropy is a function of coordination number, whereas GPC entropy is a function of root-mean square separation distance between the ends of the polymer. This can lead to improper predictions for the coarse-grained entropy. The lattice model and the GPC model both are consistent under transformations using the cross-linking entropy (CLE) model and the CLE model produce equations that include these two models with limits. The CLE model is a unifying approach to thermodynamics of biopolymers that make these models into a single framework, describing their differences and similarities, and expands the calculations and increases flexibility and incorporate important corrections such as the worm-like-chain model. CLE model is consistent with the contact order model and, when mixed with existing local pairing potentials, it can predict correct structures with minimum free energy [53].

\section{High throughput method for RNA}

As a result of advancements in high-throughput technology, the sequencing of the pioneering $16 \mathrm{~S}$ rRNA gene marker is gradually shedding light on the taxonomic characterization of the spectacular microbial diversity that inhabits the earth. 16S rRNA-based investigations of microbial environmental niches are currently conducted using several technologies, including large-scale clonal Sanger sequencing, oligonucleotide microarrays, and, particularly, 454 pyrosequencing that targets specific regions or is linked to barcoding strategies. Interestingly, the short read length produced by nextgeneration sequencing technology has led to new computational efforts in the taxonomic sequence assignment process. From a medical perspective, the characterization of the microbial composition of the skin surface, oral cavity, and gut in both healthy and diseased people enables a comparison of microbial community profiles and also contributes to the understanding of the potential impact of a particular microbial community [54]. High-throughput Omics technologies aimed at characterizing the molecular profile of diseases together with massive scientific literature on drugs and clinical trials opened the way for matching molecular profiles and drug mode of action in the realm of drug repositioning. We developed a computational analysis workflow for linking molecular targets, drugs, and diseases, and exemplified this approach for the immunosuppressive drug mycophenolate mofetil (MMF) [55]. If we look at the bibliography, history started with database building and microarray processing; both terms 'systems biology' and 'platform' in bioinformatics occur with the emergence of large databases of high-throughput data and pathways availability [56].

\section{Algorithm}

A common data mining tasks used for data categorization and analysis in industries and research is classification. Data collection deception, device restriction, data communication and analysis errors, or man-made disturbance frequently result in imprecise or vague data which is called as noisy data, which decrease the classification algorithms. Feature selection algorithm on Logistic Regression Classifier, controls False Discovery Rate (FDR) and improves its efficiency [57]. Short interfering RNAs (siRNAs) used to suppress gene expression have a lot of potential applications in therapy, but design of an effective siRNAs is still under study. Numerous siRNA design tools are ineffective as theoretically calculated property of subsequences and their efficiency for siRNA for any input set of experimental data is limited. Combining the present algorithms, a novel algorithm is derived that focuses on the stability and off-targeting to design siRNA sequences [58]. Modelling means finding a proper formulation of the problem, while solving means finding the solution of the problem. The most well-known modeling tools are: constraint programming languages, constraint libraries, (mathematical) modelling languages and specifi cation languages. Modelling languages provide the most high-level practical level of modelling for modellers [59]. In the extragenic region of genomes tandem repeats (TR) are the most abundant ones. Many have alreadyfound a large number of regulatory elements in this region. These elements show impact in the chromatin structure formation in nucleus and also contain important information in genetic evolution and phylogenic study. (http://www.trbase2. cn) [60]. New improved algorithm is more suitable for 3-D graphic environment. The first step is to break down the entire 3-D scene and then setup the Octree structure. The second step is to transform the Octree onto 2-D plane, and write down nodes which are modified and then render the modified ones. Many experiments demonstrate the superiority of the algorithm. Especially, in the process of blanking, the quadrants are shown from far to near and achieve 3-D display more effectively, according to the given object and the location of view [61]. 


\section{Viral microsatellite database}

Microsatellites are found in all known genomes are small DNA sequences with a tandem repetition of a particular motif of size 1-6 has a significant role in many fields like DNA Fingerprinting, Population Studies, Forensics, Paternity Studies, Gene Regulation, Genetic Disorder Studies, and Evolution of Genomes and also are extensively used as genetic markers for identifying pathogenic bacteria and viruses and are found to be associated with the plasticity, adaptation and virulence of bacteria and viruses. Viral Microsatellite Database (VMD), it hosts microsatellites of around 3500 viral genomes along with their alignments, locus information, imperfection info, protein info etc. The database can be accessed and downloaded for free for academic / research purposes from http://www.mcr.org.in/vmd [62].

\section{Photosensitivity}

Each protein-substrate reaction releases biophotons of light, known as autofluorescence or chemiluminescence which influences visual perception and can be adapted diagnostically. Activation and deactivation of such reaction depends on the influence of systemic parameters. Study of Physiological systems enables understanding of systemic dysfunction and pathology(s). Specific neural (EEG) frequencies associated with visual perception can be adapted therapeutically to treat specific physiological and psychological disorders [63].

\section{MatLab}

Incremental Retinal-Defocus Theory (IRDT), a homeomorphic biomechanical model of eye states that time-integrated reduction in retinal-image defocus magnitude decreases the rate of retinal neuromodulator release. The Matlab simulation results states that IRDT was able to simulate the emmetropization mechanism using only the forces acting on the masses at the nodes and neurochemical injection methods results in predicted changes in ocular deformation. This helps intherapeutic treatment to control human myopia [64].

\section{Molecular dynamics}

The HIV-1 protease enzyme produces resistance toward most of the drugs due to mutational changes but still maintains activity with their natural substrates thus is an essential target towards the HIV therapy. Docking analysis, binding energetics analysis and Molecular Dynamics simulations in absolute solvent gives an insight how the HIV-1 protease flap mutant I54M and I54L offer resistance to Darunavir upon the native HIV protease. The flap mutation I54M and I54L lowers the binding affinity of Darunavir by altering the position of binding site residues in 3D space. It decreases the electrostatic and van der Waals interaction energy and further reduction in total receptorligand interaction energy [65]. The renal artery's geometrical data from the medical texts was extracted, and pressure drops and the compliance variations of artery stenosis are applied to the electronic model. Then, the graphs were obtained for different stenotic values $(20 \%, 50 \%, 70 \%$, and $90 \%$ ) using ORCAD/MATLAB software. The cardiac pressurevolume graphs of Renal Artery Stenosis disease with different stenotic values, moreover, and are compared with normal condition. The results of stenosis show that hypertension could be a symptom of the disease. When the rate of stenosis increases the blood pressure rises. This study is a tool for students in order to see the cardiovascular system operation in normal condition or abnormal [66].

\section{Proton reactions}

The monocarboxylate transporter isoform I (MCT1, SLC16A1) expressed in Xenopus oocytes enhances catalyzing the hydration and dehydration of $\mathrm{CO}_{2}$, by a mechanism that does not require CAII catalytic activity. An explicit rate expression for the substrate flux is derived using model reduction techniques. The simulations were compared with experimental data obtained from MCT1-expressing oocytes injected with different amounts of CAII. The model suggests that CAII increases the effective rate constants of the proton reactions, possibly by working as a proton antenna [67].

\section{Frameshift genes}

A trinucleotide circular code is a set of trinucleotides allowing the reading frame in genes to be retrieved locally, i.e. anywhere in genes and in particular without start codon, and automatically with a window of a few nucleotides. The method proposed here identifies periodic signals of this code $X$ in the two frameshift types (+1 and -1$)$ of both eukaryotic and prokaryotic frameshift genes. As expected by the code theory, the circular code modulo 3 signals move in the same direction of translational frameshifting. Finally, in $68 \%$ of frameshift genes in the RECODE 2 database, the frameshift type $(+1$ and -1$)$ is automatically identified using only this circular code periodic signal. This circular code information constitutes a new structural property of frameshift genes [68].

\section{Autonomic nervous system}

Autonomic Nervous System associated with the colour perception along with the physiological systems, organs, cellular and molecular biochemistry is also associated with stress influences the stability of the physiological systems. Visual perception is influenced by levels of proteins and their reactive substrates which release biophotons, unique for their colour and level/ intensity. Thus establishing a relationship between neurosensory pathways and the autonomic nervous system and the body's function [69].

\section{Current projects}

- Development of activity and toxicity models related to antitargets

- Building a mixed docking/pharmacophore based engine for activity prediction

- Data visualization: 3D trees

\section{Conclusion}

The emerging field of Systems Biology is anticipated to have a major impact on the biosciences, moving biology from a phenomenological to a predictive science. Such predictive ability should allow to accurately foresee the outcome of therapeutic interventions with individual patients or to optimize industrial bioprocesses more precisely than has been possible before. Therefore, the results of Systems Biology are expected to have major impact on treatment and diagnosing diseases, health care and the bio-industries.

\section{References}

1. Kitano H (2002) Computational Systems Biology. Nature 420: 206-210.

2. Bu Z, Callaway DJ (2011) Proteins MOVE! Protein dynamics and long-range allostery in cell signaling. Advances in Protein Chemistry and Structural Biology 83: 163-221.

3. Kiemer L, Cesareni G (2007) Comparative interactomics: comparing apples and pears? Trends Biotechnol 25: 448-454. 
4. Anderson NL, Anderson NG (1998) Proteome and proteomics: new technologies, new concepts, and new words. Electrophoresis 19: 1853-1861.

5. Blackstock WP, Weir MP (1999) Proteomics: quantitative and physical mapping of cellular proteins. Trends Biotechnol 17: 121-127.

6. Daviss Bennett (2005) Growing pains for metabolomics. The Scientist 19: 25 28.

7. Wenk MR (2005) The emerging field of lipidomics. Nat Rev Drug Discov 4: 594-610.

8. Watson AD (2006) Thematic review series: systems biology approaches to metabolic and cardiovascular disorders. Lipidomics: a global approach to lipid analysis in biological systems. J Lipid Res 47: 2101-11.

9. Aur D, Jog MS (2010) Neuroelectrodynamics: Understanding the brain language. IOS Press

10. Singh S (2011) Membrane Permeability in Biological Systems: A Systems Biology Perspective. J Comput Sci Syst Biol 4: 027-032.

11. Prachee C, Hasnain SE (2004) Defining the Mandate of Tuberculosis Research in a Postgenomic Era. Medicinal principles and practice 13: 177-184.

12. Anandakumar S, Shanmughavel P (2008) Computational Annotation for Hypothetical Proteins of Mycobacterium Tuberculosis. J Comput Sci Syst Biol 1: 050-062.

13. Bateman A, Birney E, Cerruti L, Durbin R, Etwiller L, et al. (2002) The Pfam protein families database. Nucleic Acids Res 30: 276-80.

14. Roman L, Galperin MY, Natale DA, Koonin EV (2000) The COG database: a tool for genome-scale analysis of protein functions and evolution. Nucleic Acids Research 28: 33-36.

15. Lakshmi PTV, Uma MS, Karthikeyan PP, Annamalai A (2008) Sequence and Structure Comparison Studies of Phycocyanin in Spirulina Platensis. J Comput Sci Syst Biol 1: 063-072.

16. Oxana VG, Eugeniya ID, Igor NS (2008) Phylogenetic Analyses of the Loops in Elongation Factors EF1A: Stronger Support for the Grouping of Animal and Fungi. J Comput Sci Syst Biol 1: 073-080.

17. Shanthi V, Ramanathan K, Sethumadhavan R (2009) Role of the Cation-p Interaction in Therapeutic Proteins: A Comparative Study with Conventional Stabilizing Forces. J Comput Sci Syst Biol 2: 051-068.

18. Vijai S, Indramani, Dharmendra KC, Pallavi S (2009) HLA Class I and II Binding Promiscuity of the T-cell Epitopes in Putative Proteins of Hepatitis B Virus. J Comput Sci Syst Biol 2: 069-073.

19. Brum IJB, Martins-de-Souza D, Smolka MB, Novello JC, Galembeck E (2009) Web Based Theoretical Protein pl, MW and 2DE Map. J Comput Sci Syst Biol 2: 093-096.

20. Gao S, Wang X (2009) Predicting Type 1 Diabetes Candidate Genes using Human Protein-Protein Interaction Networks. J Comput Sci Syst Biol 2: 133146.

21. Gaur RK (2009) Prokaryotic and Eukaryotic Non-membrane Proteins have Biased Amino Acid Distribution. J Comput Sci Syst Biol 2: 298-299.

22. Taylor JM (2006) Hepatitis delta virus. Virology 344: 71-76.

23. Jones DT (1999) GenTHREADER: an efficient and reliable protein folds recognition method for genomic sequences. J Mol Biol 287: 797-815.

24. Laskowski RA, MacAurther MW, Moss DS, Thornton JM (1993) Procheck-a program to check the stereochemical quality of protein structures. J Appl Cryst 26: 47-60.

25. Singh S, Gupta SK, Nischal A, Khattri S, Nath R, et al. (2010) Comparative Modeling Study of the 3-D Structure of Small Delta Antigen Protein of Hepatitis Delta Virus. J Comput Sci Syst Biol 3: 001-004.

26. Sarker M, Chopra S, Mortelmans K, Kodukula K, Talcott C, et al. (2011) In Silico Pathway Analysis Predicts Metabolites that are Potential Antimicrobial Targets. J Comput Sci Syst Biol 4: 021-026.

27. Lakshminarasimhan N, Tagore S (2009) Damages in Metabolic Pathways: Computational Approaches. J Comput Sci Syst Biol 2: 208-215.

28. Garg N, Pundhir S, Prakash A, Kumar A (2008) PCR Primer Design: DREB Genes. J Comput Sci Syst Biol 1: 021-040.
29. Shouguo G, Shuang J, Martin H, Xujing W (2008) Quality Weighted Mean and T-test in Microarray Analysis Lead to Improved Accuracy in Gene Expression Measurements and Reduced Type I and II Errors in Differential Expression Detection. J Comput Sci Syst Biol 1: 041-049.

30. Yao L, Rongling W (2009) Modeling Host-Cancer Genetic Interactions with Multilocus Sequence Data. J Comput Sci Syst Biol 2: 024-043.

31. Pinto FR (2009) A Probabilistic Approach to Study Yeast's Gene Regulatory Network. J Comput Sci Syst Biol 2: 044-050.

32. Lutvo Kuriæ (2009) The Atomic Genetic Code. J Comput Sci Syst Biol 2: 101 116

33. Alessandro A, Di Serio C (2009) Vectors and Integration in Gene Therapy: Statistical Considerations. J Comput Sci Syst Biol 2: 117-123.

34. Sergio H, Antonio G, Juan C, Enrique Q (2009) OMICS Techniques and Identification of Pathogen Virulence Genes Application to the Analysis of Respiratory Pathogens. J Comput Sci Syst Biol 2: 124-132.

35. Manikandakumar K, Kumaran MS, Srikumar R (2009) Matrix Frequency Analysis of Oryza Sativa (japonica cultivar-group) Complete Genomes. J Comput Sci Syst Biol 2: 159-166

36. Hang X, Wu FX (2009) L1 Least Square for Cancer Diagnosis using Gene Expression Data. J Comput Sci Syst Biol 2: 167-173.

37. Pandarinath $P$, Shashi M, Rao AA (2010) Computational Study of Vira Segments Inserted within the Regions of Human Genome. J Comput Sci Syst Biol 3: 074-075.

38. Kumar GM, Prakash N, Pragya C, Krishna M (2011) Computational Analysis of SNPs in $10 \mathrm{~kb}$ Region of Human Chromosome 1. J Comput Sci Syst Biol 4 033-034.

39. Sablok G, Shekhawat NS (2008) Bioinformatics Analysis of Distribution of Microsatellite Markers (SSRs) / Single Nucleotide Polymorphism (SNPs) in Expressed Transcripts of Prosopis Juliflora: Frequency and Distribution. J Comput Sci Syst Biol 1: 087-091.

40. Hua D, Yanghua X, Wei W, Li J, Momiao X (2008) Symmetry of Metabolic Network. J Comput Sci Syst Biol 1: 001- 020.

41. Morya VK, Dewaker V, Mecarty SD, Singh R (2010) In silico Analysis of Metabolic Pathways for Identification of Putative Drug Targets for Staphylococcus aureus. J Comput Sci Syst Biol 3: 062-069.

42. Riad AM, Hassan AE, Hassan QF (2009) Investigating Performance of XML Web Services in Real-Time Business Systems. J Comput Sci Syst Biol 2: 266271 .

43. Chellapandi P, Sivaramakrishnan S, Viswanathan MB (2010) Systems Biotechnology: an Energing Trend in Metabolic Engineering of Industrial Microorganisms. J Comput Sci Syst Biol 3: 043-049.

44. Walsh MC, Nugent A, Brennan L, Gibney M J (2008) Understanding the metabolome - challenges for metabolomics. Nutrition Bulletin 33: 316-323.

45. Ragunath PK, Venkatesan P, Ravimohan R (2009) New Curriculum Design Model for Bioinformatics Postgraduate program using Systems Biology Approach. J Comput Sci Syst Biol 2: 300-305.

46. Rochfort S (2005) Metabolomics Reviewed: A New "Omics" Platform Technology for Systems Biology and Implications for Natural Products Research. J Nat Prod 68: 1813-1820.

47. Thompson MA (2004) Molecular docking using ArgusLab, an efficient shapebased search algorithm and the AScore scoring function. ACS meeting, Philadelphia 172 CINF 42 PA.

48. Kellenberger E, Rodrigo J, Muller P, Rognan D (2004) Comparative Evaluation of Eight Docking Tools for Docking and Virtual Screening Accuracy. PROTEINS Structure Function and Bioinformatics 57: 225-242.

49. Abdelouahab C, Abderrahmane B (2008) Docking Efficiency Comparison of Surflex, a Commercial Package and Arguslab, a Licensable Freeware. J Comput Sci Syst Biol 1: 081-086.

50. Vamsidhar E, Swamy GV, Chi tti S, Babu PA, Venkatasatyanarayana G, et al. (2010) Screening and Docking Studies of 266 Compounds from 7 Plant Sources as Antihypertensive Agents. J Comput Sci Syst Biol 3: 016-020. 
Citation: Siva Kishore N, Mahaboobbi S, Satya Varali M, Ramya S (2011) Emerging Trends in Various Fields with Systems Biology Approach. J Comput Sci Syst Biol S13. doi:10.4172/0974-7230.S13-004

Page 7 of 6

51. Prakash N, Patel S, Faldu NJ, Ranjan R, Sudheer DVN (2010) Molecular Docking Studies of Antimalarial Drugs for Malaria. J Comput Sci Syst Biol 3: 070- 073.

52. Adiga R, Karunasagar I, Karunasagar I (2011) Molecular Docking Studies of Type III Secretion System Effector SopB Homolog in Vibrio vulnificus. J Comput Sci Syst Biol 4: 016-020.

53. Dawson W, Kawai G (2009) Modeling the Chain Entropy of Biopolymers: Unifying Two Different Random Walk Models under One Framework. J Comput Sci Syst Biol 2: 001-023.

54. Fabrice A, Didier R (2009) Exploring Microbial Diversity Using 16S rRNA HighThroughput Methods. J Comput Sci Syst Biol 2: 074-092.

55. Mönks K, Bernthaler A, Mühlberger I, Mayer B, Oberbauer R, et al. (2011) Mycophenolate Mofetil Associated Molecular Profiles and Diseases. J Comput Sci Syst Biol 4: 001-006.

56. Turenne N (2011) Role of a Web-based Software Platform for Systems Biology. J Comput Sci Syst Biol 4: 035-041.

57. Hanuman T, Raghava NM, Siva PA, Mrithyunjaya RK, Chandra SV, et al (2009) Performance Comparative in Classification Algorithms Using Real Datasets. J Comput Sci Syst Biol 2: 097-100

58. Gupta SK, Akhoon BA, Srivastava M, Gupta SK (2010) A Novel Algorithm to Design an Efficient siRNA by Combining the Pre Proposed Rules of siRNA Designing. J Comput Sci Syst Biol 3: 005-009.

59. Rafeh R (2010) Solver Independent Modelling of Combinatorial and Optimization Problems. J Comput Sci Syst Biol 3: 086-088.

60. Liu Z, Yang Y (2009) Mining the Association Rules of Transcription Factor Binding Sites in Human Tandem Repeats Using Aprior Algorithm. J Comput Sci Syst Biol 2: 180-185.
61. Qin G, Jiang N (2010) Rendering for 3D Animation Based on Octree. J Comput Sci Syst Biol 3: 117-122.

62. Mudunuri SB, Rao AA, Pallamsetty S, Mishra P and Nagarajaram HA (2009) VMD: Viral Microsatellite Database-A Comprehensive Resource for all Vira Microsatellites. J Comput Sci Syst Biol 2: 283-286.

63. Ewing GW (2009) A Theoretical Framework for Photosensitivity: Evidence of Systemic Regulation. J Comput Sci Syst Biol 2: 287- 297

64. Hung GK, Fiedler AJ, Ciuffreda KJ (2010) Homeomorphic Matlab Model of Myopia Development. J Comput Sci Syst Biol 3: 030-039.

65. Purohit R, Rao S (2010) Effect of Flap Mutation I54L/M in Inhibition of Human Immunodeficiency Virus Type 1 Protease: Relationship to Drug Resistance. J Comput Sci Syst Biol 3: 080-085.

66. Hassani K (2010) Study of Left Ventricle Pressure-Volume Loops in Renal Stenosis Case Using Equivalent Electrical Circuit. J Comput Sci Syst Biol 3 . 076-079.

67. Almquist J, Lang P, Prätzel-Wolters D, Deitmer JW, Jirstrand M, et al. (2010) A Kinetic Model of the Monocarboxylate Transporter MCT1 and its Interaction with Carbonic Anhydrase II. J Comput Sci Syst Biol 3: 107116.

68. Ahmed A, Michel CJ (2011) Circular Code Signal in Frameshift Genes. J Comput Sci Syst Biol 4: 007-015.

69. Ewing GW, Ewing EN (2009) Does an Improved Understanding of the Nature and Structure of the Physiological Systems Lead to a Better Understanding of the Therapeutic Scope of Complementary \& Conventional Medicine? J Comput Sci Syst Biol 2: 174179. 